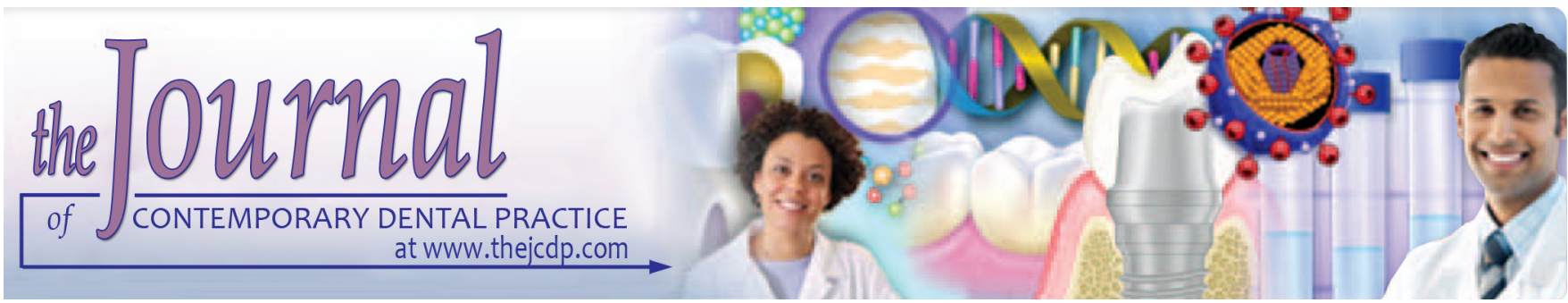

\title{
Resistin and Plasma-reactive Oxygen Metabolite Levels in Obese and Nonobese Individuals with Chronic Periodontitis in Response to Nonsurgical Periodontal Therapy
}

\author{
${ }^{1}$ Tony Varghese, ${ }^{2} \mathrm{MC}$ Prashant, ${ }^{3}$ Kiran Dodani, ${ }^{4}$ Neelu Nagpal, ${ }^{5}$ Neha Khare, ${ }^{6}$ Vikram Singh
}

\begin{abstract}
Aim: To assess and compare the impact of non-surgical periodontal therapy on plasma reactive oxygen metabolites and resistin values in chronic periodontitis obese and non-obese patients.

Materials and methods: Total 200 subjects were included in the present study and were broadly divided into two study groups with 100 patients in each group as follows: group $A$ : Obese patients with chronic periodontitis, group B: Non-obese, normal weight patients with chronic periodontitis. Various following periodontal parameter were calculated at the baseline time and two months after the nonsurgical periodontal therapy. Plasma reactive oxygen metabolite (RM) and serum and GCF resistin levelswere evaluated. Assessment of all the results was done by Statistical Package for Social Sciences (SPSS) software.
\end{abstract}

Results: Significant results were obtained while doing an intergroup comparison of clinical attachment levels between two study groups. Significant results were obtained while comparing the clinical attachment levels in both the study groups at different time intervals. Significantmki 9 reduction in the RM was seen in Group B subjects in comparison to Group A subjects 2 months after nonsurgical periodontal therapy.

Conclusion: In patients with chronic periodontitis, obesity can be considered as an important factor in the alteration of resistin levels.

\footnotetext{
1,3,5 Department of Periodontology, RKDF Dental College and Research Centre, Bhopal, Madhya Pradesh, India
}

${ }^{2}$ Department of Oral and Maxillofacial Surgery, RKDF Dental College and Research Centre, Bhopal, Madhya Pradesh, India

${ }^{4}$ Department of Oral and Maxillofacial Pathology, RKDF Dental college and Research Centre, Bhopal, Madhya Pradesh, India

${ }^{6}$ Department of Oral Medicine and Radiology, RKDF Dental College and Research Centre, Bhopal, Madhya Pradesh, India

Corresponding Author: Neelu Nagpal, Reader, Department of Oral and Maxillofacial Pathology, RKDF Dental College and Research centre, Bhopal, Madhya Pradesh, Idnia, Phone: +919754000039, e-mail: drneelu.nagpal@gamil.com
Clinical significance: Obese patients should be motivated for reducing weight so that periodontal therapy and other treatment modalities could be carried out more effectively.

Keywords: Nonsurgical periodontal therapy, Obesity, Resistin.

How to cite this article: Varghese T, Prashant MC, Dodani K, Nagpal N, Khare N, Singh V. Resistin and Plasma-reactive Oxygen Metabolite Levels in Obese and Nonobese Individuals with Chronic Periodontitis in Response to Nonsurgical Periodontal Therapy. J Contemp Dent Pract 2018;19(12):1526-1531.

Source of support: Nil

Conflict of interest: None

\section{INTRODUCTION}

Due to various implications encountered in relation to oxidative stress among late age pathologies, understanding the variations and alterations in the oxidative stress levels along with the source of alteration is a challenging task. Oxidative stress is mostly caused by reactive oxygen species (ROS). In the majority of the situations, these ROS are a by-product of energy metabolism. Hence; it justifies the directly proportional relationship of oxidative stress to energy consumption. ${ }^{1}$ Literature quotes numerous investigations that have assessed the association of systemic antioxidative status and various periodontal pathologies. ${ }^{2}$ In one of the past studies, authors reported a negative correlation between plasma vitamin $C$ levels and loss of periodontal attachment. ${ }^{3}$

In chronic periodontitis patients, lower levels of vitamin $\mathrm{E}$ and glutathione have also been reported in the past, in comparison to periodontally healthy individuals. Periodontal healthis, therefore, significantly affected by alteration in systemic antioxidative status. However; there is still paucity of data, concerning the correlation of blood ROS and periodontal condition. ${ }^{4}$

Originally discovered in 2001 in mice, Resistin (or 'resistance to insulin') got its name because of its ability 
to resist or interfere with insulin action. Soon after its discovery, it was proposed that it acts as a significant link between obesity and diabetes. White adipose tissue forms the main source of mouse resistin. A considerable increase in the mean serum concentration of resistin has also been reported in obese and diabetic patients. 5,6

Hence; under the light of data mentioned above, the present study was planned to assess the impact of nonsurgical periodontal therapy on plasma reactive oxygen metabolites and resistin values of both gingival crevicular fluid (GCF) and serum in chronic periodontitis obese and nonobese patients.

\section{MATERIALS AND METHODS}

The present investigation was commenced in the Department of Periodontology of the dental institute. Ethical approval was obtained from the of the institutional ethical committee. Along with this, written consent was also obtained from all the subjects after explaining in detail the entire research protocol.

\section{Inclusion Criteria}

- Patients within the age group of 30 to50 years

- Patients having a minimum of 20 natural teeth

- Patients with loss of clinical attachment of equal to or more than $3 \mathrm{~mm}$ in more than one-third of sites ${ }^{7}$

\section{Exclusion Criteria}

- Subjects with current pregnancy status or menopause status,

- Subjects with positive past or current smoking history,

- Subjects with presence of diabetes, hypertension or any other systemic illness

A total of 200 subjects were included in the present study and were broadly divided into two study groups with 100 patients in each group as follows:

- Group A: Obese patients with chronic periodontitis,

- Group B: Non-obese, normal weight patients with chronic periodontitis

Selection of group A subjects was done based on an assessment of waist circumference (WC) and body mass index (BMI) of the subjects, based on criteria described previously in the literature. Subjects with BMI of more than $25 \mathrm{~kg} / \mathrm{m}^{2}$ and WC of more than $90 \mathrm{~cm}$ in men and $80 \mathrm{~cm}$ in women were categorized as Obese. ${ }^{8,9}$

\section{Assessment of Parameter Clinically}

Following periodontal parameter were calculated at the baseline time:

- Gingival index (GI)

- Plaque index (PI)
- Pocket probing depth (PP)

- Loss of clinical attachment (LCA)

At the buccal and palatal sites of each tooth of the oral cavity, calculation of PI was done. Calculation of GI followed this at the proximal, labial and lingual gingival areas. Distance from the base of the periodontal pocket to the level of the cement-enamel junction was calculated and was designated as LCA. ${ }^{10}$ All the patients were called in the morning time, and from the lateral profile of the antecubital vein, a collection of blood samples was done under aseptic conditions. Two test tubes were taken; one with clot activator and the other with anticoagulant, and $1 \mathrm{~mL}$ of blood sample was collected in each of them. This was followed by immediate centrifugation at $3000 \mathrm{rpm}$ for five minutes, for separating the serum and the plasma. Estimation of resistin level in the serum was done following the method described by Furugen et al. ${ }^{11}$ Following the method described by Tamaki et al., estimation of RM levels in the plasma was done. ${ }^{12}$ The spectrophotometer was used for this. Mixing of $20 \mu \mathrm{l}$ of the chromogenic substrate was done with $20 \mu$ l of plasma sample followed by addition of $1 \mathrm{ml}$ of the prepared acetate buffer. After mixing, the prepared solution was incubated for five minutes at thirty-seven-degree centigrade. The density of the magenta color reflected the concentration of hydroperoxides within the blood. This concentration is directly proportionate to the RM quantity. Carratelli unit (CARR U) was used for measuring the RM. As per criteria previously used in the literature, $0.08 \mathrm{mg} / \mathrm{dL}$ of hydrogen peroxide is equal to one CARR U. ${ }^{10}$ As stated by Tamaki et al., categorization of RM values was done as follows: ${ }^{13}$

- Normal value: 250 to 300 CARR U

- Borderline oxidative stress: 301 to 320 CARR U

- Light oxidative stress: 320 CARR U to 340 CARR U

- Oxidative stress: 341 CARR U to 400 CARR U

- High oxidative stress: above 401 CARR U

Using a micropipette, a collection of two microliters was done at the sites with LCA of equal to more than $3 \mathrm{~mm}$ and GI score of equivalent to or more than two. Until analysis was performed, storing of the serum and GCF samples was done at minus eight-degree centigrade. Enzyme-linked immunosorbent assay (ELISA) was used for assessment of serum and GCF resistin levels. Standard curves obtained from ELISA were used for assessing the results. Measurement of the results of the GCF and serum levels was done in $\mathrm{ng} / \mathrm{mL}$.

In subjects of both the study groups, Phase I periodontal therapy was carried out. Initially, supragingival scaling was done, followed by scaling and root planning four times in 1 hour session. These proced ures were completed within two weeks' time. Baseline examination of the clinical parameters was done, including assessment of 
RM levels and serum and GCF resistin levels. Assessment of all the results was done by SPSS software. Chi-square test and Student t-test were used for assessment of the level of significance. A p-value of less than 0.05 was taken as significant.

\section{RESULTS}

In the present study, the mean age of the subjects of the group A and group B was 37.3 years and 38.4 years respectively (Table 1$)$. WC of the obese patients $(105.3 \mathrm{~cm})$ was found to be significantly higher in comparison to normal weighed subjects $(49.6 \mathrm{~cm})$. Mean BMI of the subjects of the groups A and B was 33.41 and $24.67 \mathrm{Kg} / \mathrm{m}^{2}$ respectively. Mean CLA at baseline time of the subjects of the group A and group B was 6.69 and $6.02 \mathrm{~mm}$ respectively. Mean CLA at 2 months after nonsurgical periodontal therapy of the subjects of the group A and group B was 5.23 and $4.76 \mathrm{~mm}$ respectively. Significantly results were obtained while doing an intergroup comparison of clinical attachment levels in between two study groups $(\mathrm{p}<0.05)$ (Table 2$)$. Significant results were obtained while comparing the clinical attachment levels within the groups at different time intervals ( $p$ <0.05) (Table 3). Mean Plaque index in the subjects of the group A at baseline time and 2 months after the nonsurgical therapy were 26.17 and 30.81 respectively. Among group B subjects, mean Plaque index at baseline

Table 1: Comparison of demographic details

\begin{tabular}{llll}
\hline Parameter & Group A & Group B & p-value \\
\hline Mean age (years) & 37.3 & 38.4 & 0.52 \\
Mean BMI $\left(\mathrm{Kg} / \mathrm{m}^{2}\right)$ & 33.41 & 24.67 & $0.02^{*}$ \\
WC $(\mathrm{cm})$ & 105.3 & 49.6 & $0.01^{*}$ \\
\hline
\end{tabular}

*Significant if $p<0.05$

Table 2: Intergroup comparison of clinical attachment level

\begin{tabular}{llll}
\hline Parameter & Group A & Group B & $p$-value \\
\hline CLA (mm) at baseline & 6.69 & 6.02 & $0.03^{*}$ \\
CLA after 2 months of non-surgical periodontal therapy & 5.23 & 4.76 & $0.01^{*}$ \\
\hline
\end{tabular}

*Significant if $p<0.05$

Table 3: Intragroup comparison of clinical attachment level

\begin{tabular}{lllll}
\hline Parameter & $C L A(\mathrm{~mm})$ at baseline & CLA after 2 months of non-surgical periodontal therapy & $p$-value \\
\hline Group A & 6.69 & 5.23 & $0.00^{*}$ \\
Group B & 6.02 & 4.76 & $0.00^{*}$ \\
\hline
\end{tabular}

*Significant if $p<0.05$

Table 4: Intergroup comparison of clinical parameters at different time intervals

\begin{tabular}{|c|c|c|c|}
\hline Parameter & Group A & Group B & p-value \\
\hline Plaque index at baseline & 26.17 & 34.38 & $0.02^{*}$ \\
\hline Plaque index 2 months after non-surgical periodontal therapy & 30.81 & 29.41 & 0.55 \\
\hline Gingival index at baseline & 30.15 & 30.01 & 0.67 \\
\hline Gingival index 2 months after non-surgical periodontal therapy & 34.16 & 27.68 & $0.01^{*}$ \\
\hline Probing pocket depth at baseline & 35.81 & 25.99 & $0.02^{*}$ \\
\hline Probing pocket depth 2 months after non-surgical periodontal therapy & 35.42 & 25.39 & $0.03^{*}$ \\
\hline
\end{tabular}

*Significant if $p<0.05$ 
2 months after the completion of non-surgical therapy (p-value $<0.05$ ) (Table 7). Serum resistin levels also decreased significantly 2 months after the completion of non-surgical therapy in subjects of group B. However; in obese patients, no significant alterations were seen while comparing the post-treatment serum resistin values.

\section{DISCUSSION}

One of the global epidemic problems in the present scenario, which is growing at a very rapid rate, is obesity. A positive correlation among various hormones, growth factors and bioactive compounds, such as adipokines and energy homeostasis, with the pathophysiology of obesity, has been reported in the past literature. ${ }^{14}$ Still lot of debate exists in relation to the pathophysiologic role played by resistin in human subjects. Some studies show that obese diabetic subjects have high levels of resistin mRNA expression. While on the contrary, there are some studies that show that in comparisons to the lean subjects, obese subjects had higher serum resistin level that was positively associated with changes in body mass index (BMI) and visceral fat area. ${ }^{15-17}$ It has been postulated that in obese subjects, increased susceptibility to the bacterial infections occur due to hyperoxidative state and a modified inflammatory background which causes initiation or progression of periodontal disease. oxidative stress and altered immune response because

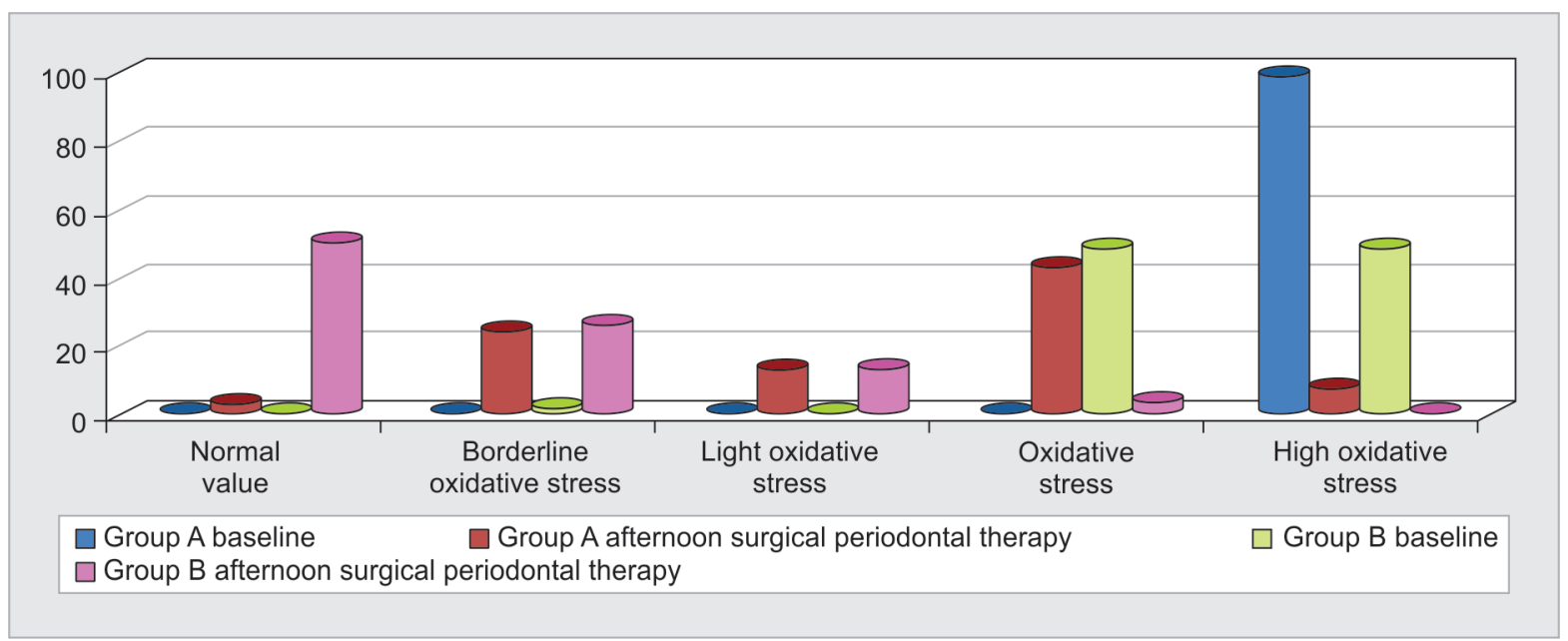

Graph 1: Alteration in RM values in the two study groups

Table 5: Intragroup comparison of clinical parameters at different time intervals

\begin{tabular}{llll}
\hline Parameter & Baseline & After 2 months of nonsurgical periodontal therapy & $p$-value \\
\hline Plaque index in Group A & 26.17 & 30.81 & $0.00^{*}$ \\
Plaque index in Group B & 34.38 & 29.41 & $0.01^{*}$ \\
Gingival index in Group A & 30.15 & 34.16 & $0.00^{*}$ \\
Gingival index in Group B & 30.01 & 27.68 & $0.00^{*}$ \\
Probing pocket depth in Group A & 35.81 & 35.42 & 0.10 \\
Probing pocket depth in Group B & 25.99 & 25.39 & 0.09 \\
\hline
\end{tabular}

*Significant if $p<0.05$

Table 6: Intergroup comparison of serum and GCF resistin levels at different time intervals

\begin{tabular}{llll}
\hline Parameter & Group A & Group B & $p$-value \\
\hline GCF resistin levels at baseline $(\mathrm{ng} / \mathrm{mL})$ & 16.02 & 13.10 & $0.00^{*}$ \\
GCF resistin levels 2 months after non-surgical periodontal therapy $(\mathrm{ng} / \mathrm{mL})$ & 13.11 & 7.54 & $0.01^{*}$ \\
Serum resistin levels at baseline $(\mathrm{ng} / \mathrm{mL})$ & 26.82 & 20.34 & $0.01^{*}$ \\
Serum resistin levels 2 months after non-surgical periodontal therapy $(\mathrm{ng} / \mathrm{mL})$ & 24.22 & 16.84 & $0.02^{*}$ \\
\hline
\end{tabular}

${ }^{*}$ Significant if $p<0.05$

Table 7: Intragroup comparison of serum and GCF resistin levels at different time intervals

\begin{tabular}{lllll}
\hline Parameter & Baseline & After 2 months of nonsurgical periodontal therapy & $p$-value \\
\hline GCF resistin levels in Group A $(\mathrm{ng} / \mathrm{mL})$ & 16.02 & 13.11 & $0.00^{*}$ \\
GCF resistin levels in group B $(\mathrm{ng} / \mathrm{mL}$ & 13.10 & 7.54 & $0.01^{*}$ \\
Serum resistin levels in group A $(\mathrm{ng} / \mathrm{mL})$ & 26.82 & 24.22 & 0.78 \\
Serum resistin levels in group B $(\mathrm{ng} / \mathrm{mL})$ & 20.34 & 16.84 & $0.00^{*}$ \\
\hline
\end{tabular}

*Significant if $\mathrm{p}<0.05$ 
of excessive adipokines might be regarded as the mechanism linking periodontal pathologies and obesity. ${ }^{18}$ Hence; under the light of data mentioned above, we planned the present investigation to assess the impact of non-surgical periodontal therapy on plasma reactive oxygen metabolites and resistin values of both gingival crevicular fluid (GCF) and serum in chronic periodontitis obese and non-obese patients.

In comparison to the normal weighed subjects (Group B), clinical attachment levels, both at baseline and 2 months after non-surgical therapy, were found to be significantly higher in obese subjects (group A). Our results were in concordance with the results obtained by Suresh $\mathrm{S}$ et al. and Suvan et al., who also reported similar findings in their study. ${ }^{10,19}$ These result highlight strong association between obesity and periodontal probing depth. In one of the previous studies conducted by Tamaki et al., the authors assessed the correlation between RM and periodontal pathologies in subjects undergoing maintenance phase of periodontal therapy. They evaluated 81 subjects with a mean age of 57.4 years, who were enrolled for a periodontal maintenance program. Examination of the PD, LCA was done. They also determined the RM and biologic antioxidant potential (BAP) levels using free radical electric evaluator. They observed the positive co rrelation of RM with mean LCA. However; PD was not found to be correlated with the percentage of LAC of equal to more than $4 \mathrm{~mm}$. They reported significantly higher levels of mean LCA in subjects with RM levels of greater than 400 CARR U in comparison to subjects with RM of equal to or less than 400 CARR U. From the results, they concluded that plasma oxidative status is positively correlated with LCA in subjects in the maintenance phase of periodontal therapy. ${ }^{13}$

In comparison to the obese subjects, a significant reduction in gingival index and LCA was observed in normal-weight subjects with chronic periodontitis (Tables 4 and 5). Similar results have been reported in the past literature by Gonçalves TE et al. and Suresh S et al., who also reported that clinical response to non-surgical periodontal therapy in impaired in obese patients with chronic periodontitis. ${ }^{10,20}$ The association between plasma oxidative status and periodontitis progression in patients undergoing maintenance phase of periodontal treatment was assessed by Machida et al. They examined a total of 45 patients with mean age of 58.8 years. Recording of PPD and LCA was done. They broadly divided the patients into two study groups; patients with low and patients with high plasma ROM levels. They observed a positive correlation between baseline plasma RM levels and mean an alteration in LCA with mean alteration in the RM levels and bleeding on probing. ${ }^{21}$
Significant results were also observed while making an intragroup comparison in relation of meaning GCF resistin levels in both the study groups at different time intervals. In inflammatory conditions such as periodontal infection, expression of resistin by inflammatory cells might be responsible for an increase in increase in resistin levels in patients with chronic periodontitis. ${ }^{22}$ Authors in one of the previous study, observed in chronic periodontitis patients, that improvement in periodontal inflammation and plasma RM values occur during non-surgical periodontal treatment therapy. Their findings indicated that elevated plasma RM levels may contribute to alteration in periodontal conditions. ${ }^{12,13}$ Significant results were obtained while comparing serum and GCF levels among subjects of groups A and $B$ at different time intervals. However; in obese patients, no significant alterations were seen while comparing the post-treatment serum resistin values. The results obtained were in correlation with the results obtained by Suresh et al., who also reported similar findings in their study. Comparable findings were also reported in the past literature by Gonçalves et al. who proposed that obesity might have a modulatory effect on the resistin levels, which further may lead to the generation of systemic and local pro-inflammatory response in obese subjects with periodontitis. ${ }^{20}$ Suresh et al. comparatively evaluated the plasma RM levels in obese subjects with healthy and inflammatory periodontal status and concluded that oxidative stress is comparatively higher in obese subjects with periodontitis in comparison to obese subjects with healthy periodontium. ${ }^{23}$ Therefore, as evident from the above data, in subjects with chronic periodontitis, obesity can be considered as a modifying parameter of resistin levels.

\section{CONCLUSION}

In obese subjects, significant reduction in the plasma RM and GCF resistin levels occurred. Also, these were found to be significantly correlated with clinical periodontal parameters in obese patients with chronic periodontitis. Reduction in periodontal inflammation and therefore ROS occurs in patients undergoing nonsurgical periodontal therapy. Hence; further risk of the development of systemic diseases is reduced by it. However, further studies are recommended.

\section{REFERENCES}

1. Beamonte-Barrientos R, Verhulst S. Plasma reactive oxygen metabolites, and non-enzymatic antioxidant capacity are not affected by an acute increase of metabolic rate in zebra finches. J Comp Physiol B. 2013 Jul;183(5):675-83.

2. Amarasena N, OgawaH, Yoshihara A,Hanada N,MiyazakiH. Serum vitamin C-periodontal relationship in communitydwelling elderly Japanese. J Clin Periodontol 2005;32:93-97. 
3. Amaliya, Timmerman MF, Abbas F, Van der Weijden GA, Van Winkelhoff AJ, Winkel EG, et al. Java project on periodontal diseases: The relationship between vitamin $C$ and the severity of periodontitis. J Clin Periodontol 2007;34:299-304.

4. Panjamurthy K, Manoharan S, Ramachandran CR. Lipid peroxidation and antioxidant status in patients with periodontitis. Cell Mol Biol Lett 2005;10:255-264.

5. Jamaluddin MS, Weakley SM, Yao Q, Chen C. Resistin: functional roles and therapeutic considerations for cardiovascular disease. British Journal of Pharmacology. 2012;165(3):622-632.

6. Gerber M, Boettner A, Seidel B, Lammert A, Bar J, Schuster $\mathrm{E}$, et al. Serum resistin levels of obese and lean children and adolescents: biochemical analysis and clinical relevance. J Clin Endocrinol Metab. 2005;90:4503-4509.

7. Armitage GC. Development of a classification system for periodontal diseases and conditions. Ann Periodontol 1999;4:1-6.

8. Physical status: The use and interpretation of anthropometry. Report of a WHO expert committee. World Health Organ Tech Rep Ser 1995;854:1-452

9. Alberti KG, Zimmet P, Shaw J. Metabolic syndrome-A new world-wide definition. A Consensus statement from the international diabetes federation. Diabet Med 2006;23:469-480

10. Suresh S, Mahendra J, Singh G, Pradeep Kumar AR, Thilagar $\mathrm{S}$, Rao N. Effect of nonsurgical periodontal therapy on plasma-reactive oxygen metabolite and gingival crevicular fluid resistin and serum resistin levels in obese and normal weight individuals with chronic periodontitis. J Indian Soc Periodontol 2018;22:310-316

11. Furugen R, Hayashida H, Yamaguchi N, Yoshihara A, OgawaH, Miyazaki $\mathrm{H}$, et al. The relationship between periodontal condition and serum levels of resistin and adiponectin in elderly Japanese. J Periodontal Res 2008;43:556-562.

12. Tamaki N, Tomofuji T, Ekuni D, Yamanaka R, Yamamoto T, Morita $\mathrm{M}$, et al. Short-term effects of non-surgical periodontal treatment on plasma level of reactive oxygen metabolites in patients with chronic periodontitis. J Periodontol 2009;80: 901-906.

13. Tamaki N, Tomofuji T, Maruyama T, Ekuni D, Yamanaka R, Takeuchi N, et al. Relationship between periodontal condition and plasma reactive oxygen metabolites in patients in the maintenance phase of periodontal treatment. J Periodontol 2008;79:2136-2142.
14. Nieva-Vazquez A, Pérez-Fuentes R, Torres-Rasgado E, LópezLópez JG, Romero JR. Serum Resistin Levels Are Associated with Adiposity and Insulin Sensitivity in Obese Hispanic Subjects. Metabolic Syndrome and Related Disorders. 2014;12(2):143-148.

15. McTernan PG, McTernan CL, Chetty R, Jenner K, Fisher FM, Lauer MN.. Increased resistin gene and protein expression in human abdominal adipose tissue. J Clin Endocrinol Metab 2002; $87: 2407-2410$

16. Heilbronn LK, Rood J, Janderova L, Albu JB, Kelley DE, Ravussin E, et al. Relationship between serum resistin concentrations and insulin resistance in nonobese, obese, and obese diabetic subjects. J Clin Endocrinol Metab 2004;89: 1844-1848

17. Vendrell J, Broch M, Vilarrasa N, Molina A, Gómez JM, Gutiérrez C, et al. Resistin, adiponectin, ghrelin, leptin, and proinflammatory cytokines: Relationships in obesity. Obes Res 2004;12:962-971.

18. Waddington RJ, Moseley R, Embery G. Reactive oxygen species: A potential role in the pathogenesis of periodontal diseases. Oral Dis 2000;6:138-151.

19. Suvan JE, Petrie A, Nibali L, Darbar U, Rakmanee T, Donos N, et al. Association between overweight/obesity and increased risk of periodontitis. J ClinPeriodontol 2015;42:733-739.

20. Gonçalves TE, Feres M, Zimmermann GS, Faveri M, Figueiredo LC, Braga PG, et al. Effects of scaling and root planing on clinical response and serum levels of adipocytokines in patients with obesity and chronic periodontitis. J Periodontol 2015;86:53-61.

21. Machida T, Tomofuji T, Ekuni D, Yamane M, Yoneda T, Kawabata Y et al. Longitudinal Relationship between Plasma Reactive Oxygen Metabolites and Periodontal Condition in the Maintenance Phase of Periodontal Treatment. Dis Markers. 2014;2014:41-49.

22. Patel SP, Raju PA. Gingival crevicular fluid and serum levels of resistin in obese and non-obese subjects with and without periodontitis and association with single nucleotide polymorphism at-420. J Indian Soc Periodontol 2014;18:555-559.

23. Suresh S1, Mahendra J, Sudhakar U, Pradeep AR, Singh G. Evaluation of plasma reactive oxygen metabolites levels in obese subjects with periodontal disease. Indian J Dent Res. 2016 Mar-Apr;27(2):155-159. 